\title{
CONTEMPORARY CBRN (CHEMICAL, BIOLOGICAL, RADIOLOGICAL, NUCLEAR) THREATS AND ADEQUATE RESPONSE - REAL SITUATION TRAINING
}

\section{Boris RAJČIĆ ${ }^{*}$, Gvozden TASIĆ ${ }^{* *}$, Vladimir KARIĆ ${ }^{* * *}$, Bojan RADAK $^{* * * *}$, Dubravka MILOVANOVIĆ}

\begin{abstract}
Testing and training in the field of chemical, biological, and radiation detection, for decontamination and protection against radiological agents, toxic chemicals and biological agents, are emerging security fields. They are gaining significance due to the increasing number of events involving the use of these substances in public places. Indoor (laboratory) and outdoor testing and training processes are both necessary for developing an adequate response and adequate procedures. Testing is required for decontamination equipment and agents, as well as for decontamination process efficiency. It is important to investigate and compare the effects of real outdoor conditions and standard laboratory conditions. Periodical trprljavainings are crucial for enabling first-responders to prevent or respond to and recover from the full spectrum of chemical, biological, radiological and nuclear threats. Various scenarios for outdoor trainings are being developed in order to make the trainings as realistic as possible. For example, these scenarios may include small quantities of pyrotechnics as simulators of dirty bombs when the detection of the scattered material in the environment is the subject of training. Also, the trainings can include toxic materials found in the environment as a result of accidents, natural disasters, misuse, as well as radiological emitters (open and sealed). The safety strategy during the trainings includes using highly effective protective clothing and equipment. Trainings in realistic conditions and with live agents, even proxies, are generally regarded as the only way to

${ }^{*}$ MSc, Research Assistant,Vinca Institute of Nuclear Sciences, University of Belgrade, Belgrade, Serbia, boris@vinca.rs

${ }^{* *}$ Research Assistant, Vinca Institute of Nuclear Sciences, University of Belgrade, Belgrade, Serbia, gvozden.tasic@vin.bg.ac.rs

${ }^{* * *}$ MSc, Research assistant ,Vinca Institute of Nuclear Sciences, University of Belgrade, Belgrade, Serbia,ppz@vinca.rs

${ }^{* * * *}$ Science Advisor Vinca Institute of Nuclear Sciences, University of Belgrade, Belgrade, Serbia, bradak@vin.bg.ac.rs

${ }^{* * * * *} \mathrm{PhD}$, Vinca Institute of Nuclear Sciences, University of Belgrade, Belgrade, Serbia, duca@vinca.rs
\end{abstract}


fully prepare first-responders and other specialists for adequate procedures, the stress level and decision-making capabilities in cases of high-risk events.

Keywords: CBRN agents, training, detection, decontamination, PPE

\section{INTRODUCTION}

In recent years, the number of terrorist attacks involving toxic and deadly agents has been increasing. They underscore the magnitude and importance of emergency response procedures in cases of events that include chemical, biological, radiological, or nuclear (CBRN) agents. Besides the situations where CBRN agents are released as an act of war or terrorism, there are everyday situations where accidents involving toxic materials can occur. To ensure a proper reaction of first-responders (police, firefighters, the military, explosive specialists, medical personnel), it is necessary to develop and constantly improve specific training and testing programs which can provide realistic threat training, while remaining within safety and legislative boundaries (Cekovic, et al., 2004; Fatah, et al., 2001; Yang, et al., 1992). Testing and training includes the detection of agents, as well as the use of personal protection equipment, sampling procedures (if needed), and decontamination procedures (Bodurtha \& Dickson, 2016). Testing and training should include a theoretical and practical part. The theoretical part is delivered in classrooms, and the practical part is delivered both indoors, in a laboratory, and outdoors, at specialized sites. Besides their significance for the training of first-responders, these practical indoor and outdoor activities are important for the evaluation and comparison of the detection and decontamination effects between outdoor conditions and standard laboratory conditions (Šutulović \& Ceković, 2004; Guzman, et al., 2016).

\section{CHARACTERISTICS AND TYPES OF CBRN AGENTS}

Hazardous chemical substances have the potential to harm or destroy living tissue or disrupt vital processes, leading to incapacitation, illness or death. Of all CBRN substances, the chemical ones are the most common, due to their ubiquity in the supply chain, economy and everyday life. Besides Chemical Warfare Agents (CWA), there are other toxic chemicals and explosive precursors, the most common of which are Toxic Industrial Materials (TIM). Some TIMs are: ammonia, chlorine, cyanogen chloride, hydrogen cyanide, phosgene, etc. (Ghosh, et al., 2010; Sandor, 2014; Fatah, et al., 2001).

It is common knowledge that ionizing radiation is dangerous to health and life due to the changes it causes in cell metabolism, disrupting the functioning of an organism and sometimes leading to death. There are two types of ionizing radiation: electromagnetic radiation (gamma, X-ray, and UV radiation) and corpuscular radiation (alpha and beta particles, protons, neutrons, and fragments of heavy atomic nuclei) (Cekovic, et al., 2004; Kumar, et al., 2010). Radiological agents have an impact on deoxyribonucleic acid (DNA) itself, which causes disorder in DNA synthesis i.e. has a detrimental effect on blood, reproductive organs, and young cells.

Biological agents have many ways of causing mass casualties due to their high lethal potential, which is generally higher than that of chemical agents. The equipment and means 
for producing bio-agents are fully available on the civilian market (even from internet shops), and are not always sold under the supervision of relevant security services. Bioweapons are easy to hide and colourless and odourless, unlike chemical weapons. Biological agents include pathogenic bacteria, fungi, viruses, and biological toxins. These agents can cause fatal or chronic illness, leading to epidemics, mass panic, and the disruption of social order ( $\mathrm{Lu}$, et al., 2010). The Centers for Disease Control and Prevention (CDC) classifies biological agents into three categories: A, highest-priority pathogens (Bacillus anthracis, Clostridium botulinum toxin, Variola mayor, filoviruses-ebola), B, second highest-priority bio-agents (Brucella species, Salmonella species, Escherichia coli, Chlamydia psittaci, Ricin), and C, emerging pathogens that could be engineered for mass dissemination (Nipah virus, Hantavirus, Yellow fever virus, Multi-drug-resistant tuberculosis) (Cekovic, et al., 2004; Chomiczewski, 2003; Masthan, et al., 2012).

Nuclear weapons are by far the most dangerous weapons on Earth. They can jeopardize the natural environment and the lives of future generations on a long-term basis, destroy cities and even countries, killing millions of people. Fortunately, they have only been used twice in warfare. The best protection against such catastrophic weapons is disarmament; a number of multilateral treaties have been established with the aim of preventing nuclear proliferation and testing (United Nations Office for Disarmament Affairs, 2018).

\section{TRAININGS FOR CBRN THREATS}

It is possible to efficiently enable first-responders to adequately prevent, prepare for or respond to, and recover from the full spectrum of CBRN threats if they undergo trainings with real or proxy CBRN agents.

When designing a training course, it is important to consider the entry knowledge of the trainees. For example, Vinča Institute offers different difficulty levels of CBRN trainings using entry tests to determine the appropriate level. The type of training can also vary the course needs to be adapted to each specialist group of first-responders.

The basic-level CBRN general and safety awareness training is a classroom course which familiarizes the trainees with CBRN materials, their respective properties and the measures that can be taken for protection. CBRN detection training covers detection techniques for the agents. In CBRN decontamination training, the participants master decontamination techniques. Advanced live agent trainings, CBRN forensics, and scenario trainings are intended for participants with considerable entry knowledge, since those trainings cover analyses of CBRN agent incidents to determine the magnitude of the problem, sampling and operating as a group within a live CBRN environment. Training with live agents and in realistic situations is invaluable in evaluating the behaviour and real capabilities of individuals and groups.

In responder training for CBRN threats, the responders must become familiar with all aspects of CBRN activities. The theoretical part of the training should provide them with knowledge of CBRN agents and their physiochemical properties (colour, odour, density, ways of entering the human body, etc.). Knowing the types of decontamination equipment and the categories of Personal Protective Equipment (PPEs) is mandatory. All theoretical 
knowledge should be enacted in realistic situations in the practical part of the course. The scenarios should include some simulators of dirty bombs, e.g. small quantities of pyrotechnics; simulators of scattered material in the environment; contaminated objects from everyday life (seats, vehicles, suitcases, etc.).

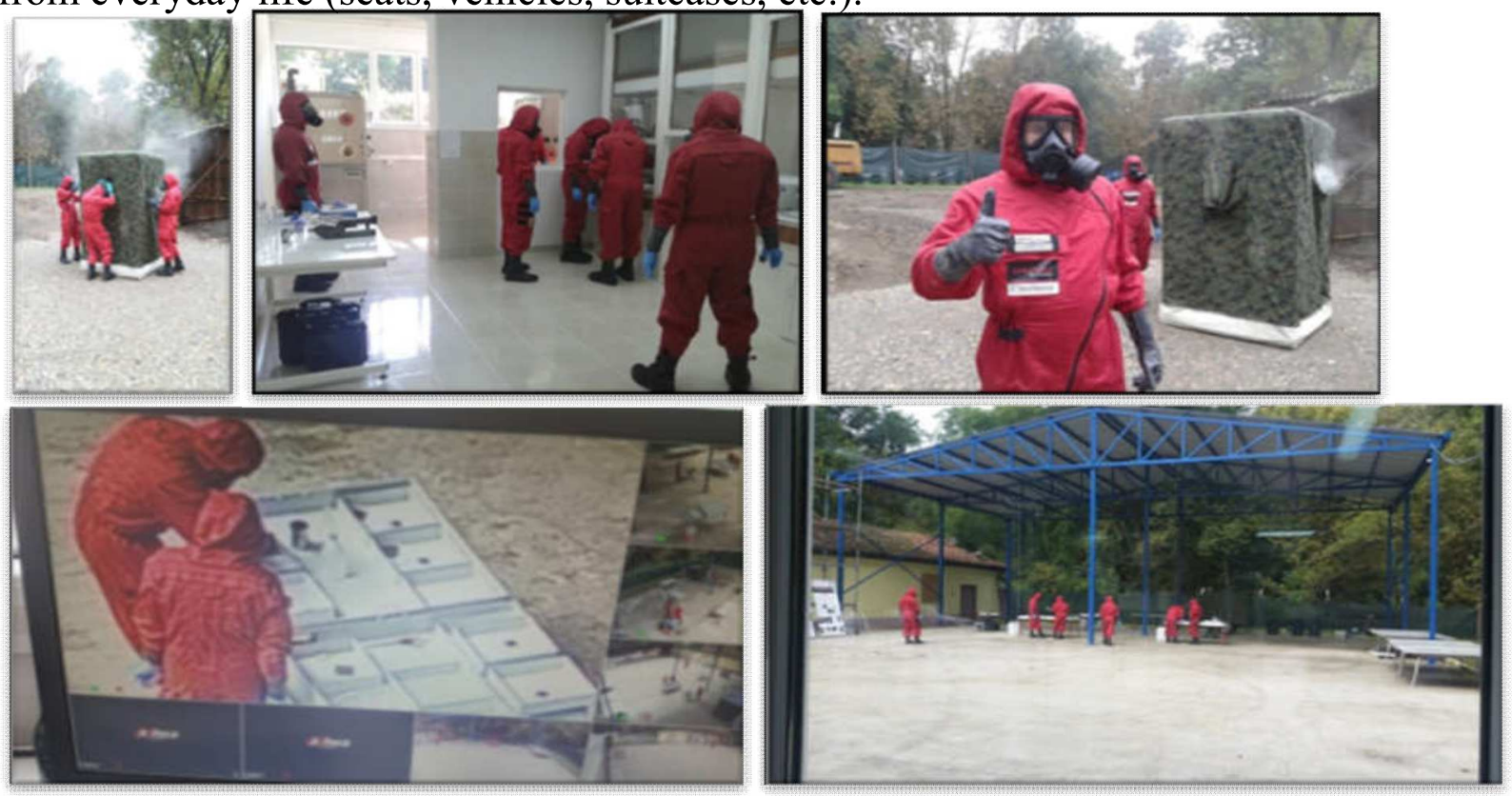

After the trainees decontaminate the scenario area, the most important part of the training is removing the PPEs in the right way and correct order. The concept of three zones can be applied, depending on the level of the CBRN hazard and contamination: the hot zone, where significant contamination with CBRN agents has been confirmed or is strongly suspected, but not characterized, and presumed to be life threatening (both skin contact and inhalation); the warm zone, where contamination is possible but active release has ended and initial monitoring exists; the cold zone, where contamination is unlikely - this zone covers the area beyond the expected significant dispersal range of the initial event and the secondary contamination range caused by traffic and emergency responders. When the detection and decontamination in the hot zone are done, the undressing process starts. This process has several stages. First, special equipment (detection instruments, decontamination fitment, communications, etc.) is put aside and deposed in protective shield containers. The process of assembling the equipment and undressing the trainees must be supervised by a safety officer. Next comes decontamination and the disposing of the PPE during the transit from the hot to the warm zone. The PPE must be decontaminated in a manner deemed appropriate for the identified hazard (biocide preparation, water, decontamination solution, detergent). The disposal order is: coveralls half way up $\rightarrow$ overboots $\rightarrow$ gloves $\rightarrow$ all of the coveralls. In CBRN operations, using protective clothing is required, with at least two pairs of gloves, of which the inner gloves are removed at the end. The mask with a filter should be removed from the face as late as possible. This is usually done during the transit from the warm to the cold zone. Without enough practice or on impulse, trainees may remove the mask at the beginning of the undressing process, exposing the person to a facial skin infection or the inhalation of a 
dangerous agent. The preparation and implementation of decontamination in a safe way requires repeated training and exercise.

The trainees get in contact with detection equipment/instruments, PPE, and practical decontamination procedures and techniques. Here, we outline the protective equipment and decontamination activity only.

Because of the limited capacity of human senses to detect CBRN agents, detection and identification is supported by instruments or appropriate sets. Detection is not just realizing that there is a threat, but also locating it with more or less precision, depending on the instrument. There are many instruments and detection kits for the rapid detection and identification of chemical agents.

First-responders sent to a potential CBRN contamination area must be provided with accurate up-to-date information. The organizers must assess the risk of a hazardous substance release and the conditions present, instruct the responders, and base Personal Protective Equipment (PPE) selection on that knowledge. Two basic aspects are of significance: respiratory protection and skin (dermal) protection. Based on these, the PPE is a set comprising a respiratory mask and a personal protective ensemble. Personal protective clothing typically consists of: an over-garment - protection for the body (torso, arms, legs), footwear - protection for the feet (overboots on top of regular footwear), and gloves - protection for the hands (made from air-impermeable materials or butyl rubber gloves and butyl rubber gloves additionally coated with neoprene).

At low hazard levels, air-permeable protective clothing selection is suitable. To protect the hands, gloves made of air- and liquid-impermeable materials are mandatory. High CBRN hazard levels, i.e. severe liquid contamination, may dictate the use of a PPE suit based on impermeable materials. An alternative to a non-ventilated impermeable protective suit is based on a simple, lightweight, disposable air- and liquid-impermeable material with an absorptive liner underneath. This concept solves the problems of decontamination, the effect of pinholes, bad closures and occlusion. When only radioactive dust hazard exists, using a full-face respirator, gloves, disposable overboots and lightweight disposable dustimpermeable coverall wear on top of regular working clothing is sufficient (Holland \& Cawthon, 2014).

\section{DECONTAMINATION OF CBRN AGENTS}

Decontamination is simply the removal of the contamination agent by mechanical or chemical means or by dilution.

Mechanical methods are the simplest and fastest to implement; removing the outerwear results in reducing the pollution by $85-90 \%$. It should be implemented in the case of mass casualties. The chemical way is to neutralize the toxic effect of a hazardous substance by changing its chemical structure to a new, non-hazardous or less hazardous one. A biological agent can be neutralized by its chemical destruction (Holland \& Cawthon, 2014; Oudejans, et al., 2016). The most common method of decontamination is dilution reducing the concentration of the hazardous substance by scattering it into a thinner one. In the contaminated area, the fastest available thinner is water. In practice, this is done 
using a water jet or a so-called decontamination booth. This is also the most economical way of execution. However, in most cases, the use of water does not alter the composition of a hazardous substance and only lowers the concentration. The usual decontamination solutions for chemical decontamination are: $10 \% \mathrm{Ca}(\mathrm{ClO})_{2}$ (calcium hypochlorite), $5 \%$ $\mathrm{Na}_{2} \mathrm{CO}_{3}$ (sodium carbonate), $5 \% \mathrm{Na}_{3} \mathrm{PO}_{4}$ (sodium phosphate), $1 \% \mathrm{HCl}$ (hydrochloric acid), a water solution with detergent (Cekovic, et al., 2004; Amitai, et al., 2010; Sutulović \& Ceković, 2004).

\section{CONCLUSION}

Today's CBRN threats are becoming more probable, more complex, and are developing both in type and ways of occurrence. Staying up-to-date with all of their aspects is vital. These threats come not only from war-related activities, but also arise in everyday life situations in accidents involving toxic or harmful materials. They are now omnipresent, in industry and also in civilian life. Methods of harmful agent detection therefore need constant improvement. Testing is required for protection and decontamination equipment, as well as for decontamination process efficiency.

Realistic situation experience outdoors is invaluable, both in training and in testing the methods and equipment:

- Comparing laboratory results with effects in outdoor real situations;

- Testing the efficiency of detection and decontamination methods in the field;

- Using realistic scenarios, with live agents (real or proxy) as the key to fully preparing personnel for operations, stress levels, and decision-making in a CBRN event;

- Revealing important flaws in individual or group preparedness so that they can be rectified (psychological adequacy, group support, group cohesion and synchronization, etc.);

- Tailoring the trainings is important, so that they are most effectively adopted by groups of different compositions and levels of knowledge, experience, purpose, etc.

\section{REFERENCES}

United Nations Office for Disarmament Affairs. (2018). Retrieved from United Nations: https://www.un.org/disarmament/wmd/nuclear/

Amitai, G., Hironobu, M., Andersen, J., Koepsel, R., \& Russell, A. (2010). Decontamination of chemical and biological warfare agents with a single multifunctional material. Biomaterials, 31(15), 4417-4425.

Bodurtha, P., \& Dickson, E. (2016). Decontamination science and Personal Protective Equipment (PPE) selection for Chemical-Biological-Radiological-Nuclear (CBRN) events. Alberta: Defence Research and Development Canada.

Cekovic, B., Mladenovic, V., Lukovic, Z., Karkalic, R., \& Krstic, D. (2004). Comparative Research on Chemical, Radiological and Biological Decontamination Efficiency of Present Decontaminants and of Multipurpose Emulsion-Based Decontaminant (in Serbian). Belgrade: Scientific Technical Information, Military Technical Institute. 
Chomiczewski, K. (2003). The bioterrorism threat. Epidemiological review, 57(2), 349353.

Fatah, A., Barrett, J., Arcilesi, R., Ewing, K., Lattin, C., Helinski, M., et al. (2001). Guide for the Selection of Chemical and Biological Decontamination Equipment for Emergency First Responders. Washington, DC: DIANE Publishing.

Ghosh, T., Prelas, M., Viswanath, D., \& Loyalka, S. (2010). Science and Technology of Terrorism and Counterterrorism (2nd ed.). Boca Raton: CRC Press Taylor \& Francis Group.

Guzman, R., Navaro, R., Ferre, J., \& Moreno, M. (2016). RESCUER: Development of a Modular Chemical, Biological, Radiological, and Nuclear Robot for Intervention, Sampling, and Situation Awareness. Journal of Field Robotics, 33(7), 931-945.

Holland, M., \& Cawthon, D. (2014). Personal protective equipment and decontamination of adults and children. Emergency Medicine Clinics of North America, 33(1), 5168.

Kumar, V., Goel, R., Chawla, R., Silambarasan, M., \& Sharma, R. (2010). Chemical, biological, radiological, and nuclear decontamination: Recent trends and future perspective. Journal of Pharmacy \& Bioallied Sciences, 2(3), 220-238.

Lu, T., Yiao, S., Lim, K., Jensen, R., \& Hsiao, L. (2010). Interpretation of biological and mechanical variations between the Lowry versus Bradford method for protein quantification. North American Journal of Medical Sciences, 2(7), 325-328.

Masthan, K., Shanmugam, K., Aravindha, B., \& Tathagata, B. (2012). Virus as a biological-weapon. International Research Journal of Microbiology, 2(6), 237239.

Oudejans, L., O'Kelly, J., Evans, A., Wyrzykowska-Ceradini, B., Touati, A., Tabor, D., et al. (2016). Decontamination of Personal Protective Equipment and Related Materials Contaminated with Toxic Industrial Chemicals and Chemical Warfare Agent Surrogates. Journal of Environmental Chemical Engineering, 4(3), 27452753.

Sandor, S. (2014). Latest technologies suitable for chemical decontamination of sensitive equipment and interior for the Hungarian defence forces. Katonai Technical Scineces, 130-136.

Šutulović, L., \& Ceković, B. (2004). Overview of Perspectives for the Destruction of Toxic Chemicals and CWA According to the Chemical Weapons Convention. 4th International Conference of the Chemical Societies of the South-East European Countries on Chemical Sciences in Changing Times: Visions, Challenges and Solutions. II, p. 148. Belgrade: Serbian Chemical Society.

Yang, Y., Baker, J., \& Ward, R. (1992). Decontamination of Chemical Warfare Agents. Chemical Review, 92, 1729-1743. 